\title{
Short-term treatment outcomes and dropout risk in males and females with eating disorders
}

Zaida Agüera ${ }^{\mathrm{a}, \mathrm{b}, \mathbb{I}^{*}}$; Isabel Sánchez ${ }^{\mathrm{b} \mathscr{I}}$; Roser Granero ${ }^{\mathrm{a}, \mathrm{c}}$; Nadine Riesco ${ }^{\mathrm{b}}$; Trevor Steward ${ }^{\mathrm{a}, \mathrm{b}}$; Virginia Martín-Romera ${ }^{\mathrm{d}}$; Susana Jiménez-Murcia ${ }^{\mathrm{a}, \mathrm{b}, \mathrm{e}}$ Mariarita Caroleo ${ }^{\mathrm{f}}$; Cristina SeguraGarcía $^{\text {f,g }}$; José Manuel Menchon ${ }^{\text {bee,h }}$; Fernando Fernández-Aranda ${ }^{\text {a,b,e* }}$

${ }^{a}$ CIBER Fisiología de la Obesidad y Nutrición (CIBERobn), Instituto Salud Carlos III, Madrid, Spain

${ }^{b}$ Department of Psychiatry, University Hospital of Bellvitge-IDIBELL, Barcelona, Spain.

${ }^{c}$ Departament de Psicobiologia $i$ Metodologia. Universitat Autònoma de Barcelona, Barcelona, Spain

${ }^{d}$ Departament de Psicologia Clínica i Salut. Universitat Autònoma de Barcelona, Barcelona, Spain

${ }^{e}$ Department of Clinical Sciences, School of Medicine, University of Barcelona, Barcelona, Spain

${ }^{f}$ Department of Health Sciences, University Magna Graecia, Catanzaro, Italy

${ }^{g}$ Ambulatory for Clinical Research and Treatment of Eating Disorders, University Hospital Mater Domini, Catanzaro, Italy

${ }^{h}$ CIBER Salud Mental (CIBERSAM), Instituto Salud Carlos III, Barcelona, Spain

${ }^{\text {II }}$ shared first authorship

*Corresponding authors:

Fernando Fernández-Aranda, Ph.D., FAED, Department of Psychiatry, University Hospital of Bellvitge-IDIBELL and CIBEROBN, c/ Feixa Llarga s/n, 08907 - Barcelona, Spain (email: fendo@wanadoo.es; Tel. +34932607227; fax.+34932607193).

This is the author manuscript accepted for publication and has undergone full peer review but has not been through the copyediting, typesetting, pagination and proofreading process, which may lead to differences between this version and the Version of Record. Please cite this article as doi: 10.1002/erv.2519

This article is protected by copyright. All rights reserved. 
Zaida Agüera, Ph.D., Department of Psychiatry, University Hospital of Bellvitge-IDIBELL and CIBEROBN, c/ Feixa Llarga s/n, 08907 - Barcelona, Spain (e-mail: zaguera@bellvitgehospital.cat; Tel. +34932607227).

\begin{abstract}
This study compared treatment outcomes between males and females with eating disorders (ED) and analyzed clinical predictors of treatment outcome. Our sample consisted of 131 male and 131 female ED patients who underwent cognitive-behavioral therapy (CBT) treatment. ED severity, personality and psychopathology were assessed using standard instruments. We found that the risk of dropout was higher for males with $\mathrm{BN}$ than for females with $\mathrm{BN}$, and that males with $\mathrm{BN}$ and OSFED were more likely to obtain full remission in comparison with their female counterparts. Predictive models of treatment outcome indicated that higher scores in novelty seeking was a shared factor associated with higher risk of dropout and not obtaining full remission for both men and women with ED. However, only in men, younger age and lower scores in reward dependence predicted higher dropout. Contrastingly, higher persistence scores were predictors of full remission. This study reinforces the effectiveness of using outpatient CBT as treatment as usual for men with ED. Nonetheless, placing greater emphasis on strategies targeting gender specific issues could enhance outcomes.
\end{abstract}


Keywords: Cognitive-behavioral therapy, dropout, eating disorders, gender, treatment outcome.

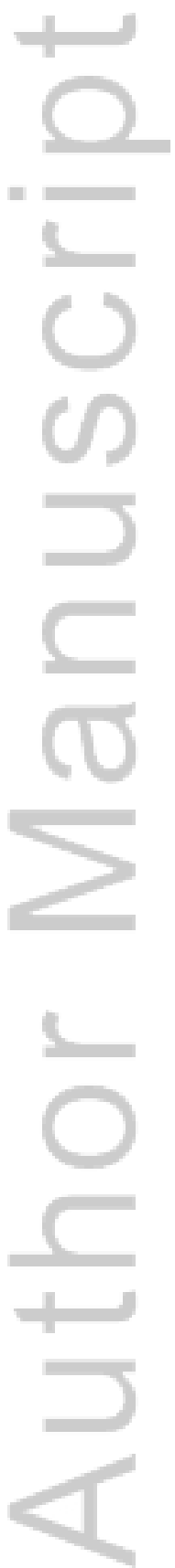

This article is protected by copyright. All rights reserved. 


\section{Introduction}

Clinical and epidemiological studies on eating disorders (ED) have highlighted the lower prevalence of males with these disorders in comparison with females (approximately one male for every nine females with ED) (Fernández-Aranda \& JiménezMurcia, 2014; Ulfvebrand, Birgegård, Norring, Högdahl, \& von Hausswolff-Juhlin, 2015). However, these data could be underestimated since ED are often considered as a 'female phenomenon', and this stigma might lead to males with ED being under-diagnosed and to seeking treatment with less frequency (Griffiths et al., 2015; Strother, Lemberg, Stanford, \& Turberville, 2012). This could explain why this topic has received relatively little attention from researchers, and why the few studies focusing on ED in males and treatment outcome have had such small sample sizes (Fernández-Aranda \& Jiménez-Murcia, 2014; Thapliyal \& Hay, 2014).

Although men and women with ED share similar clinical characteristics, some gender particularities have been described. Regarding personality traits, several studies have revealed lower levels of harm avoidance, reward dependence, cooperativeness, and higher scores in novelty seeking in men with ED than women with the same disorder (Fernández-Aranda et al., 2004; Núñez-Navarro et al., 2012; Woodside et al., 2004). Men with ED also show less concern about weight and body concerns (Núñez-Navarro et al., 2012; Shingleton, Thompson-brenner, Thompson, Pratt, \& Franko, 2015; Shu et al., 2015), as well as greater general psychopathology (Weltzin et al., 2005). However, these characteristics may be reflective of socio-cultural gender differences, as these differential patterns are similar to those described between males and females in healthy, non-ED populations (Fernández- 
Aranda \& Jiménez-Murcia, 2014; Núñez-Navarro et al., 2012). Despite this, some specific features of men with ED have been observed. Within these, it should be noted that men with ED more frequently identify as being gay, use distinct compensatory behaviors (e.g. men present higher frequency of vomiting episodes but less laxative abuse than women), are more likely to have a history of premorbid overweight or obesity, later age of ED onset, carry out more physical activity, and have a higher mortality risk than ED women (Fernández-Aranda et al., 2004; Gueguen et al., 2012; Mitchison \& Mond, 2015; Núñez-Navarro et al., 2012).

In addition, if there is a paucity of literature focusing on the clinical features of ED males, studies comparing treatment outcome between men and women with ED are even scarcer. To date, treatment approaches are often based on therapies developed for women with ED, and male-specific treatment guidelines are not available. The few published studies on treatment in males with ED have focused mainly on anorexia nervosa (AN) patients undergoing residential/inpatient treatment (Weltzin, Bean, Klosterman, Lee, \& WelkRichards, 2015), and relatively little attention has been paid to men with other ED diagnoses, such as bulimia nervosa $(\mathrm{BN})$, binge eating disorder (BED) or eating disorders not otherwise specified (EDNOS) undergoing outpatient group treatment (Fernández-Aranda \& JiménezMurcia, 2014). The few studies assessing treatment outcome in men with AN reported better outcomes for males than for females (Bean et al., 2008; Strober et al., 2006). Studies focusing on treatment outcome in men with BN or EDNOS reveal contradictory findings. While some studies found similar outcomes for males and females with $\mathrm{BN}$, as well as a similar risk of dropout (Fernández-Aranda, Krug, et al., 2009; Weltzin et al., 2015), Stoving et al. (Støving, Andries, Brixen, Bilenberg, \& Hørder, 2011) identified better outcomes for males than for 
females. However, all these studies were limited in sample size (samples of 5, 8 or 19 men with $\mathrm{BN}$, respectively).

Taking into account all the aforementioned gaps in the literature, mainly contradictory findings and studies where males represented a negligible portion of the sample, it is not possible to generalize results or define a clear hypothesis about treatment outcome in males with ED. Therefore, the aims of the present study were the twofold: 1) to compare treatment outcome (remission and risk of dropout) in a large sample of men and women with eating disorders, in which different ED diagnoses, defined according to the DSM-5 criteria (APA, 2013), were represented; and 2) to assess specific and differential predictors of treatment outcome in men and women with ED. The results of this study may contribute to advancements in the knowledge on whether men with ED equally benefit from treatment as usual [cognitive-behavioral therapy (CBT)] as women. 


\section{Material and Methods}

\section{Participants}

Our sample comprised every male patient, over 18 years of age, who was consecutively admitted for treatment at the Eating Disorders Unit at Bellvitge University Hospital between 1998 and 2015. From a total sample of 157 men who were diagnosed with an eating disorder, 26 were excluded from the study because they refused treatment (final sample $n=131$ males with ED). This group of men with ED was compared with 131 female ED patients, matched for age and DSM-5 diagnosis. Patients were matched using SPSS software (SPSS Inc., Chicago, IL, USA). Each case from the male ED group was paired to a randomly selected female ED patient from a larger pool of female ED cases from our Unit. No statistical differences were found comparing selected and unselected women for this study sample in sociodemographic variables, baseline state or treatment outcome.

All patients admitted before May of 2013 were originally diagnosed using the Diagnostic and Statistical Manual for Mental Disorders (DSM-IV-TR) (APA, 2000). 
Diagnoses were made by means of a semi-structured clinical interview (Fernández-Aranda \& Turón, 1998), based on the validated SCID-I interview (First, Spitzer, Gibbon, \& Williams, 2002), and conducted by experienced clinical psychologists and psychiatrists. Diagnoses were reanalyzed post hoc using DSM-5 criteria (APA, 2013). According to DSM-5 criteria, the final sample was composed of 56 restrictive anorexia nervosa (AN-R) patients, 22 bingepurging AN (AN-BP) patients, $80 \mathrm{BN}, 28 \mathrm{BED}$, and 76 Other Specified Feeding and Eating Disorders (OSFED) patients. Each group was equally made up of males and females. Patients were excluded from the analysis if they presented medical or nutritional complications and/or severe co-morbid psychopathology (i.e. suicide attempts) requiring hospitalized inpatient treatment. Non-responder patients (mainly severe AN patients who did not regain weight during the treatment) were hospitalized. These patients were only included in the study after their inpatient treatment, and only if they continued treatment at the day hospital.

In accordance with the Declaration of Helsinki, the present study was approved by the Ethics Committee of our institution [The Clinical Research Ethics Committee (CEIC) of Bellvitge University Hospital], and all the participants provided signed informed consent.

\section{Measures}

Eating Disorder Inventory-2 (EDI-2 (Garner, 1991)). This is a reliable and valid 91item multidimensional self-report questionnaire that assesses different cognitive and behavioral characteristics of eating disorders: drive for thinness, body dissatisfaction, bulimia, ineffectiveness, perfectionism, interpersonal distrust, interoceptive awareness, maturity fears, asceticism, impulse regulation, and social insecurity. This instrument was 
validated in a Spanish population (Garner, 1998). Internal consistency was excellent in our sample $(\alpha=.95)$.

Symptom Checklist-90 Items-Revised (SCL-90-R (Derogatis, 1994)). This is a 90-item questionnaire widely used for assessing self-reported psychological distress and psychopathology. The test is scored on nine primary symptom dimensions: somatization, obsessive-compulsive, interpersonal sensitivity, depression, anxiety, hostility, phobic anxiety, paranoid ideation and psychoticism, and three global indices: Global Severity Index (GSI), Positive Symptom Total (PST), and Positive Symptom Distress Index (PSDI). This instrument was validated in a Spanish population (Derogatis, 2002). Internal consistency was excellent in our sample $(\alpha=.98)$.

Temperament and Character Inventory-Revised (TCI-R (Cloninger, 1999)). The TCI$\mathrm{R}$ is a 240 -item questionnaire with a five-point Likert scale format. This questionnaire is a reliable and valid measure of four temperaments (harm avoidance, novelty seeking, reward dependence and persistence) and three character dimensions (self-directedness, cooperativeness and self-transcendence) of personality. This questionnaire has been validated in a Spanish adult population (Gutiérrez-Zotes et al., 2004). Cronbach's alpha for the current sample was good ( $\alpha=.79$ for "novelty seeking") to excellent ( $\alpha=.89$ for "harm avoidance", "persistence" and "self-directedness") (see supplementary Table S1).

Motivational scale. Motivational state was assessed through a visual analogue scale, including five different questions which evaluated: the subjective severity of the disorder, perceived need for treatment; social impairment of daily tasks, the level of worry the patient had over their disorder (self-concern), and the extent of which the patient thought their 
relatives were worried (family-concern). The scales ranged from 0 to 8 , with 8 being the maximum score indicating worry and motivation for change. This scale has previously been described and applied in ED patients (Casanovas et al., 2007)

\section{$\underline{\text { Treatment }}$}

Every patient with AN (both men and women) received the same day hospital (DH) treatment that covered nutritional-dietary patterns and psychological-psychiatric aspects, based on a CBT program previously described elsewhere (Fernández-Aranda \& Turón, 1998), with demonstrated effectiveness (Agüera et al., 2015). Patients attended the DH from 9 a.m. to 3 p.m., five days a week (Monday - Friday), for a period of 15 weeks. Food intake was monitored twice during breakfast and lunch (the main food intake of the day).

Treatment for the other ED diagnoses, such as BN, OSFED and BED, consisted of 16 weekly manualized outpatient group therapy sessions lasting 90-minutes each (FernándezAranda \& Turón, 1998) and based on Fairburn et al. (Fairburn, Marcus, \& Wilson, 1993) model. This program and accompanying material have already been manualized and published in Spanish (Fernández-Aranda \& Turón, 1998) with demonstrated effectiveness (Agüera et al., 2013). Men and women were also treated in different groups. Following the guidelines of previous studies, males were treated in a setting of "male-only" group therapy (Fernández-Aranda, Krug, et al., 2009; Weltzin et al., 2012), where several male-specific topics were emphasized (e.g. stigmatization, sexual orientation, and concern about sex drive (libido) decrease due to a lowering of testosterone levels), as described elsewhere (Fernández-Aranda \& Jiménez-Murcia, 2014; Fernández-Aranda, Krug, et al., 2009). 
Patients were re-evaluated at discharge and categorized into three categories: "full remission", "partial remission", and "non-remission". Voluntary treatment discontinuation was categorized as "dropout" (i.e. not attending treatment for three consecutive sessions was considered dropout). These categories were based on the consensus judgment of the senior clinical staff who considered all aspects of the patient's treatment outcome, such as normalization of nutritional-dietary patterns, frequency of binge episodes and compensatory behaviors (such as self-induced vomiting or laxative and diuretics misuse), weight restoration, improvement in attitudes regarding weight and shape, and ED cognitions. To assess clinical significance of response to treatment, the therapists had access to objective primary measures, such as weight recovery and the number of binging/purging episodes per week, based on entries from a food diary. Following the guidelines of treatment outcome according to DSM-5 criteria (APA, 2013), the working definition of a "full remission" outcome was a total absence of symptoms meeting diagnostic criteria for at least 4 consecutive weeks, "partial remission" was defined as substantial symptomatic improvement but the continued presence of residual symptoms for at least 4 consecutive weeks, and the patients who presented poor outcomes were defined as "non-remission". These categories were previously used to assess treatment outcome in other published studies (Agüera et al., 2013, 2015; Custal et al., 2014; Sauchelli et al., 2016; Steward et al., 2016).

As a complementary therapy outcome, the reliable change index (RCI) defined by Jacobson and Truax (1991) was used. This definition is obtained for each participant by dividing the pre- and post-therapy change by the standard error of the difference between the two scores/measures. This measure constitutes a dimensional score into the theoretical range 
of $-\infty$ to $+\infty$. On the basis that RCI values larger than 1.96 would be unlikely to occur $(p<.05)$ without real therapy changes, it is usual to obtain a binary classification of low-RCI ( $\mathrm{RCI}<1.96$, interpreted as lack of improvement after the therapy) versus high-RCI ( $\mathrm{RCI} \geq 1.96$, interpreted as improvement after treatment). In this study, the RCI scores were generated for three measures: BMI, the frequency of binges and the frequency of vomits. To consider improvement after treatment based on the RCI, the ED subtype was considered: a) for AN, therapy improvement was considered for participants who achieved RCI $\geq 1.96$ in BMI; b) for $\mathrm{BN}$, therapy improvement was considered for $\mathrm{RCI} \geq 1.96$ in both, the frequency of vomits and binges; c) for BED, therapy improvement was considered for $\mathrm{RCI} \geq 1.96$ in the frequency of binges; and d) for OSFED, therapy improvement was considered for $\mathrm{RCI} \geq 1.96$ in any of the three indexes (BMI, binges or vomits).

\section{Statistical analysis}

Statistical analysis was carried out with SPSS20 for Windows. Comparison between men and women at baseline for categorical variables was carried out with chi-square $\left(\chi^{2}\right)$ tests procedure and with t-tests for quantitative variables. Multinomial logistic regressions compared CBT outcome between men and women, including the EDI-2 total score as covariate. Given that men and women with EDs showed significant differences in ED severity (EDI-2 total score) (see Table 1), the statistical analyses were adjusted for this variable in order to guarantee that the results were not best explained for the differences in ED severity. Separate models were obtained for ED diagnoses (AN, BN, BED and OSFED). 
Survival analysis stratified by diagnosis modeled the time until the dropout during the therapy. In this analysis, the Kaplan-Meier (KM) estimated the cumulate $\mathrm{S}(\mathrm{t})$ probability that a patient survives without dropping out longer than a time $t$. Comparison of survival functions between men and women was carried out through Cox regression adjusted for the covariate EDI-2 total score.

- Stepwise logistic regression in two steps-blocks generated predictive models for the primary therapy outcomes dropout and full remission, separately for women and men. Models were not adjusted for diagnosis due to the low frequency for the outcomes considered in this study. In the first step, diagnosis was entered and set. The second step automatically selected through stepwise procedure the best significant predictors, defining the patients' chronological age, onset of the ED, body mass index (BMI), ED severity (EDI-2 total score), global psychopathological state (SCL-90R global indexes) and personality (TCI-R scores) in the list of potential predictors. The goodness-of-fit for the final selected models was assessed by the Hosmer-Lemeshow test ( $p>.05$ was considered adequate fit), the global predictive capacity with the Nagelkerke's pseudo- $\mathrm{R}^{2}$ coefficient and the global discriminative capacity with the area under the ROC (AUC) curve.

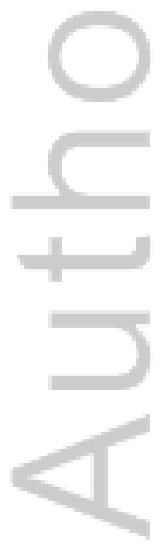




\section{Results}

Descriptive for the sample at baseline

Table 1 includes a description of the sample at baseline (before the beginning of treatment), with sociodemographic and ED-related variables. No statistical differences between men and women were found, except for in the frequency of laxative use (higher mean for women, 2.18 weekly vs. $0.61 ; p=.011$ ) and in overall ED severity (higher means on the EDI-2 total score for women, $97.6 v s .74 .8 ; p<.001)$.

\section{--- Insert Table 1 about here ---}

Table S1 (supplementary) includes the frequency distribution of the psychometric scales, internal consistency (Cronbach's-alpha coefficients) in the own sample, and a comparison by gender. Regarding motivational state, our results just showed statistical differences in social impairment of daily tasks and in self-concern, with women with ED presenting higher scores on these two dimensions [(5.17 ( $\mathrm{SD}=2.28)$ vs. $4.34(\mathrm{SD}=2.76) ; p=.011$ and $6.46(\mathrm{SD}=1.84)$ vs. $5.87(\mathrm{SD}=2.23) ; p=.026$, respectively).

Comparison of treatment outcomes between men and women 
Of the patients who completed therapy, the percentage of subjects who obtain full remission was $56.25 \% .31 .77 \%$ reached partial remission and $11.98 \%$, non-remission. Table 2 includes the distribution of therapy outcomes for the total sample (including the participants who dropped out, as an intent-to-treat analysis) stratified by gender and ED diagnosis [Table S2 supplementary- contains the distribution stratified by sex, ED-diagnosis and therapy type (day-hospital versus outpatient therapy), as well as the distribution for therapy outcome classification based on the RCI criterion]. A first multinomial regression model indicated that the interaction between sex-by-CBT type was statistically non-significant (Likelihood ratio chi-square LR- $\chi^{2}=1.67, p=.664$ ), allowing the estimation of main effects considering simultaneously the day-hospital and outpatient subsamples. However, since the interaction sex-by-ED diagnosis was significant $\left(\mathrm{LR}-\chi^{2}=29.1, p=.001\right)$, the comparison between men and women was obtained separately for each ED group (AN, BN, BED and OSFED). After adjusting for ED severity (EDI-2 total score), statistical differences between men and women were found for the patients diagnosed with $\mathrm{BN}\left(\mathrm{LR}-\chi^{2}=36.2, p<.001\right.$, with the risk of dropout and full remission higher for men compared to women) and OSFED (LR- $\chi^{2}=169.5, p=.008$, with the risk of dropout higher for women and the risk of full remission higher for men).

\section{--- Insert Table 2 about here ---}

Results were quite similar considering the RCI therapy outcome criterion (Table S3, supplementary): differences between men and women also emerged for $\mathrm{BN}$ and OSFED subsamples.

Figure 1 includes the KM plot for the survival time to the dropout during the therapy. The $\mathrm{x}$ axis represents the time (in weeks) when treatment began to the end of the intervention. The 
y-axis corresponds to the proportion of patients' surviving without dropping out. At time zero, $100 \%$ of the patients are alive without an event (i.e. no dropout was registered). A solid line displays the events, that is, a register of each dropout. After adjusting for ED severity (EDI-2 total score), no statistical differences between men and women were found in the cumulate survival functions within the AN, BED and OSFED subsamples. Statistical differences were found for $\mathrm{BN}$ subsample, in the sense that rate to dropout was higher for men compared to women (specifically, the KM-plots indicate that for men and women all the dropouts occurred during the first 8 weeks the first half of the treatment program, but dropouts for women occurred at a lower rate than men).

\section{--- Insert Figure 1 about here ---}

\section{Predictive models for primary treatment outcomes: dropout and full remission}

Table S4 (supplementary) contains the distribution of the potential predictors of the main therapy outcomes considered in the study, separately for men and women: dropout, full remission, low-RCI and high RCI. Table S5 (supplementary) contains the distribution of pre to post-clinical changes in the BMI, the frequency of binges and the frequency of vomits.

Table 3 includes the final binary predictive models of the primary treatment outcomes: dropout and full remission, obtained via stepwise logistic regression procedures (Table S3supplementary- includes the means and standard deviations of the potential predictors of these primary outcomes). Results, adjusted for the covariate of ED diagnosis, indicated that higher scores in novelty seeking predicted higher risk of dropout and lower risk of full remission for both women and men. In the male group, higher risk of dropout was also predicted by younger age, and lower levels of reward dependence, while higher risk of full 
remission was predicted by higher scores in persistence. All the models included in Table 3 achieved goodness-of-fit ( $p>.05$ in the Hosmer-Lemeshow tests). Global predictive and discriminative capacity was excellent for the models adjusted for women, and very good for the models adjusted for men.

The models for the criterion RCI (Table S6, supplementary) showed that, adjusted for ED diagnosis, high changes for men were related to low scores in the novelty seeking trait $(\mathrm{OR}=0.98, p=.049)$, while no significant predictor was retained for women (the only quasisignificant contributor was high scores in the reward dependence dimension $(\mathrm{OR}=1.03$, $p=.091)$.

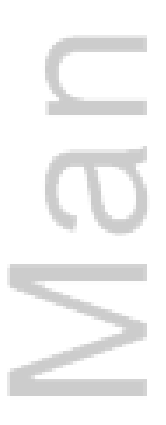

\section{Discussion}

In an attempt to gain better insight into the treatment of men with $\mathrm{ED}$, this study evaluated similarities and differences regarding treatment outcomes and dropout rates in men and women with ED (using DSM-5 diagnostic criteria). This study also examined differential predicting factors between sexes. It aimed to provide information that could be useful in the revision of ED treatment guidelines for men, a minority in the field of ED that must not be overlooked. 
Our first main finding was that, although both gender groups obtained positive results from treatment, male ED patients who completed treatment (specifically those with $\mathrm{BN}$ and OSFED) presented higher full remission rates than females with the same diagnosis. These findings are in line with prior research (Bean et al., 2008; Støving et al., 2011; Strober et al., 2006), but they are in disagreement with other studies indicating similar outcomes for male and female with ED (Fernández-Aranda, Krug, et al., 2009; Gueguen et al., 2012; Weltzin et al., 2015). These inconsistencies may be due to the fact that many previous studies were limited by small sample power when considering males with ED. Also, since most studies have assessed patients in residential/inpatient treatment settings, their findings might be slightly inflated due to increased comorbidity and symptom severity in men who need intensive treatment. Contrastingly, our findings also indicate that males with $\mathrm{BN}$ had higher dropout rates in comparison with female BN patients. An intriguing finding, however, was that those male and female patients that managed to reach the halfway point in the treatment program no longer dropped out. From a clinical perspective, and according to our results, it could be suggested that treatment discontinuation might be associated with a lower level of motivation (less perception of social impairment and less self-concern). Similarly, the fact that dropouts for $\mathrm{BN}$ men occurred at a higher rate than $\mathrm{BN}$ women suggests that low motivation in males might be linked with the stigma surrounding ED as a 'female phenomenon' and therefore, it might be a salient barrier for adherence to treatment and reluctance to seek treatment, (Griffiths et al., 2015; Strother et al., 2012).

In terms of primary predictors, we found that some clinical and personality characteristics were associated with treatment outcome. Our findings indicated that the trait 
novelty seeking was strongly associated with treatment outcome in both men and women with ED. Higher scores in novelty seeking (namely the tendency to be more impulsive, excitable, dramatic, and intolerant of routine) were related to higher dropout risk, as well as worse prognosis in both genders. This is in line with prior research showing that greater impulsivity and novelty seeking were associated with a higher risk of therapy failure, treatment resistance, a lower remission rate and dropout in females with ED (Castellini et al., 2012; Fernández-Aranda, Álvarez-Moya, et al., 2009; Halmi, 2013). These findings suggest that more impulsive patients, both males and females, would benefit from alternative interventions that successfully reduce impulsivity. Such interventions could potentially enhance treatment adherence and overall therapeutic results (Fernández-Aranda et al., 2015; Giner-Bartolomé et al., 2015). However, if we consider the results obtained using the RCI (clinical significant change) criterion, novelty seeking does not list as predictor of improvement in women with ED. This discrepancy between high RCI and "full remission" could be justified by the fact that presenting a high RCI does not imply remission, just symptomatological improvement (i.e. a patient with $\mathrm{BN}$ who started treatment presenting 21 binges/vomits per week and ended treatment with 5 binges/vomits per week would present a high RCI, but she would be in the partial remission group instead of the full remission. The same would occur for a patient with AN who started treatment with a very low BMI and regained a significant amount of weight, but not enough to reach a BMI of over 18 , then she would be considered as a high RCI but only in partial remission). Moreover, our findings also indicate that both younger age and lower scores in reward dependence were associated with higher dropout risk in the group of males with ED. The lack of treatment adherence in males 
with these characteristics is not surprising given that patients with low reward dependence have been described as seeking little emotional support, having a poor disposition to being sociable, and having difficulty in expressing their feelings and thoughts (Cloninger, Svrakic, \& Przybeck, 1993). Likewise, higher scores in persistence were positively associated with full remission. Being that persistence has been defined as having perseverance despite frustration and fatigue (Cloninger et al., 1993), this finding might explain why more persistent male patients are more likely to carry out treatment promoting changes.

\section{Limitations and Strength}

The present study should be evaluated within the context of its several limitations. First, we included only adult ED patients from Spain. Hence, we cannot confirm whether our results are generalizable to adolescent ED patients or to individuals from other ethnic backgrounds. Second, the questionnaires used to assess eating and shape concepts were designed for women, therefore further evaluations of body image concerns for men are needed to improve the validity and reliability of these scales for males. Third, the present study did not use the same standardized questionnaires at the end of the treatment (as objective measures), which would have contributed to the reliability and objectivity of our longitudinal findings. Fourth, this study is limited by the lack of follow-up data. Hence, the results have to be interpreted cautiously as we only assessed the participants at posttreatment, and there is no way of knowing the extent to which these effects persisted over time. Our findings only allow us to talk about response during treatment or symptomatological remission, but not recovery (i.e. the term "recovery" requires a long period of abstinence from ED symptomatology, and therefore follow-up data). Future 
longitudinal studies should aim to collect this information and to replicate this study in order to assess whether there is a differential rate of relapse. Finally, no predictive models were obtained for the risk of dropout and full remission stratified by the diagnosis, due the very low frequency of these outcomes in some diagnostic subsamples. Future research should also validate the predictors obtained in this work considering ED diagnoses separately.

Notwithstanding these limitations, the current study has also several strengths. For the first time, we have addressed treatment response and dropout rates across a large sample of men with ED, including different DSM-5 diagnoses. The results also add to a growing body of research examining predictors of treatment outcome in ED patients receiving CBT therapy. As far as we know, this is the first study assessing predictors of treatment outcome in ED males. Past studies of treatment outcome in men ED have included many ED diagnoses, though limited sample size in these cases did not allow for analyzing specific predictive models of outcomes.

\section{Conclusions}

In conclusion, our findings indicate that, even though outpatient CBT treatment for ED appears to be effective for both genders, male patients who complete the therapy (specifically those with $\mathrm{BN}$ or OSFED) benefit more from this type of intervention than female patients with the same diagnosis (Støving et al., 2011). Furthermore, lower scores in novelty seeking appeared to be related to overall improvement and to a lesser risk of dropout in both men and women with ED. Also, specific predictors of poor outcome were found just in males, namely being younger and obtaining lower scores in persistence and reward dependence. In light of our results, this study reinforces arguments of continuing to use 
gender-based treatment as usual for men with ED (Greenberg \& Schoen, 2008). That said, placing greater emphasis on strategies that promote motivation, treatment adherence, persistence and reward dependence, as well as decreasing impulsivity could potentially enhance results in this group. Also, the concept of stigmatization should be addressed in the first sessions of therapy to lessen refusal of treatment.

\section{Acknowledgements}

Funding: This work was supported by Instituto de Salud Carlos III (ISCIII) [grant number FIS PI14/00290 and European Regional Development Fund (ERDF)-"a way to Build Europe"] and by a grant of the Ministerio de Economía y Competitividad (grant number PSI2015-68701-R). Centro de Investigación Biomédica en Red Fisiopatología de la Obesidad y Nutrición (CIBERobn) and Centro de Investigación Biomédica en Red Salud Mental (CIBERSAM) are both initiatives of ISCIII.

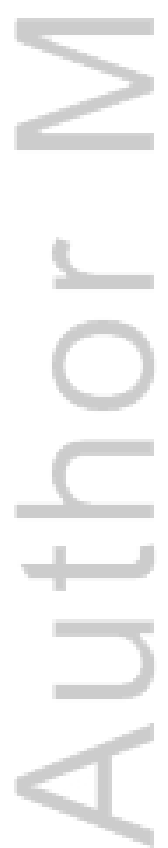




\section{References}

Agüera, Z., Riesco, N., Jiménez-Murcia, S., Islam, M. A., Granero, R., Vicente, E., ...

Fernández-Aranda, F. (2013). Cognitive behaviour therapy response and dropout rate across purging and nonpurging bulimia nervosa and binge eating disorder: DSM-5 implications. BMC Psychiatry, 13, 285. doi:10.1186/1471-244X-13-285

Agüera, Z., Romero, X., Arcelus, J., Sánchez, I., Riesco, N., Jiménez-Murcia, S., ... Fernández-Aranda, F. (2015). Changes in Body Composition in Anorexia Nervosa: Predictors of Recovery and Treatment Outcome. PLoS One, 10(11), e0143012.

APA. (2000). Diagnostic and Statistical Manual of Mental Disorders: DSM-IV-TR. New York American Psychiatric Press Inc (Vol. 4th). doi:10.1176/appi.books.9780890423349.5847

APA. (2013). Diagnostic and statistical manual of mental disorders: DSM-5. Washington, DC: American Psychiatric Association.

Bean, P., Welk, R., Hallinan, P., Cornella-Carlson, T., Weisensel, N., \& Weltzin, T. E. (2008). The Effects of a Multidisciplinary Approach to Treatment in the Recovery of 
Males and Females Diagnosed with Anorexia Nervosa in the Presence and Absence of Co-Morbid Obsessive Compulsive Disorders. Journal of Groups in Addiction \& Recovery, 3(3-4), 305-321. doi:10.1080/15560350802424969

Casanovas, C., Fernández-Aranda, F., Granero, R., Krug, I., Jimenez-Murcia, S., Bulik, C. M., \& Vallejo-Ruiloba, J. (2007). Motivation to change in eating disorders: clinical and therapeutic implications. European Eating Disorder Review, 15(6), 449-456.

Castellini, G., Mannucci, E., Lo Sauro, C., Benni, L., Lazzeretti, L., Ravaldi, C., ... Ricca, V. (2012). Different moderators of cognitive-behavioral therapy on subjective and objective binge eating in bulimia nervosa and binge eating disorder: a three-year follow-up study. Psychotherapy and Psychosomatics, 81(1), 11-20. doi:10.1159/000329358

Cloninger, C. R. (1999). The Temperament and Character Inventory-Revised. St Louis, MO: Center for Psychobiology of Personality, Washington University.

Cloninger, C. R., Svrakic, D. M., \& Przybeck, T. R. (1993). A psychobiological model of temperament and character. Archives of General Psychiatry, 50(12), 975-990.

Custal, N., Arcelus, J., Agüera, Z., Bove, F., Wales, J., Granero, R., ... Fernandez-Aranda, F. (2014). Treatment outcome of patients with comorbid type 1 diabetes and eating disorders. BMC Psychiatry, 14, 140. doi:10.1186/1471-244X-14-140

Derogatis, L. R. (1994). SCL-90-R: Symptom Checklist-90-R. Administration, Scoring and Procedures Manuall-II for the revised version. Towson; MD: Clinical Psychometric Research. 
Derogatis, L. R. (2002). SCL-90-R. Cuestionario de 90 síntomas-Manual. Madrid: TEA Editorial.

Fairburn, C. G., Marcus, M. ., \& Wilson, G. (1993). Cognitive-behavioral therapy for binge eating and bulimia nervosa: A comprehensive treatment manual. In C. G. Fairburn \& G. T. Wilson (Eds.), Binge eating: Nature, assessment and treatment (pp. 361-404). New York: Guilford Press.

Fernández-Aranda, F., Aitken, A., Badía, A., Giménez, L., Solano, R., Collier, D., ... Vallejo, J. (2004). Personality and psychopathological traits of males with an eating disorder. European Eating Disorders Review, 12(6), 367-374. doi:10.1002/erv.603

Fernández-Aranda, F., Álvarez-Moya, E. M., Martínez-Viana, C., Sànchez, I., Granero, R., Penelo, E., ... Peñas-Lledó, E. (2009). Predictors of early change in bulimia nervosa after a brief psychoeducational therapy. Appetite, 52(3), 805-808. doi:10.1016/j.appet.2009.03.013

Fernández-Aranda, F., \& Jiménez-Murcia, S. (2014). Evidence-Guided Treatment for Males with Eating Disorders. In I. Dancyger \& V. Fornari (Eds.), Evidence Based Treatments for Eating Disorders: Children, Adolescents and Adults (pp. 203-218). New York: Nova Publishers.

Fernández-Aranda, F., Jimenez-Murcia, S., Santamaría, J. J., Giner-Bartolomé, C., MestreBach, G., Granero, R., ... Menchó, J. M. (2015). The Use of Videogames as Complementary Therapeutic Tool for Cognitive Behavioral Therapy in Bulimia Nervosa 
Patients. Cyberpsychology, Behavior and Social Networking, 18(12), 744-751. doi:10.1089/cyber.2015.0265

Fernández-Aranda, F., Krug, I., Jiménez-Murcia, S., Granero, R., Núñez, A., Penelo, E., ... Treasure, J. (2009). Male eating disorders and therapy: A controlled pilot study with one year follow-up. Journal of Behavior Therapy and Experimental Psychiatry, 40(3), 479486. doi:10.1016/j.jbtep.2009.06.004

Fernández-Aranda, F., \& Turón, V. (1998). Trastornos alimentarios. Guia basica de tratamiento en anorexia y bulimia. Barcelona: Masson.

First, M. B., Spitzer, R. L., Gibbon, M., \& Williams, J. B. W. (2002). Structured Clinical Interview for DSM-IV-TR Axis I Disorders, Research Version, Patient Edition. (SCIDI/P). New York: Biometrics Research, New York State Psychiatric Institute.

Garner, D. M. (1991). Eating Disorder Inventory-2. Odessa: Psychological Assessment Resources.

Garner, D. M. (1998). Inventario de Trastornos de la Conducta Alimentaria (EDI-2)-Manual. Madrid: TEA.

Giner-Bartolomé, C., Fagundo, A. B., Sánchez, I., Jiménez-Murcia, S., Santamaría, J. J., Ladouceur, R., ... Fernández-Aranda, F. (2015). Can an intervention based on a serious videogame prior to cognitive behavioral therapy be helpful in bulimia nervosa? A clinical case study. Frontiers in Psychology, 6(July), 1-9. doi:10.3389/fpsyg.2015.00982 
Greenberg, S. T., \& Schoen, E. G. (2008). Males and eating disorders: Gender-based therapy for eating disorder recovery. Professional Psychology: Research and Practice, 39(4), 464-471. doi:10.1037/0735-7028.39.4.464

Griffiths, S., Mond, J. M., Li, Z., Gunatilake, S., Murray, S. B., Sheffield, J., \& Touyz, S. (2015). Self-stigma of seeking treatment and being male predict an increased likelihood of having an undiagnosed eating disorder. International Journal of Eating Disorders, 48(6), 775-778. doi:10.1002/eat.22413

Gueguen, J., Godart, N., Chambry, J., Brun-Eberentz, A., Foulon, C., Divac, S. M., .. Huas, C. (2012). Severe anorexia nervosa in men: Comparison with severe AN in women and analysis of mortality. International Journal of Eating Disorders, 45(4), 537-545. doi:10.1002/eat.20987

Gutiérrez-Zotes, J. A., Bayón, C., Montserrat, C., Valero, J., Labad, A., \& Cloninger, C. R. (2004). Temperament and Character Inventory-Revised (TCI-R). Standardization and normative data in a general population sample. Actas Españolas de Psiquiatria, 32(1), $8-15$.

Halmi, K. A. (2013). Perplexities of treatment resistence in eating disorders. BMC Psychiatry, 13, 292. doi:10.1186/1471-244X-13-292

Jacobson, N. S., \& Truax, P. (1991). Clinical significance: a statistical approach to defining meaningful change in psychotherapy research. Journal of Consulting and Clinical Psychology, 59(1), 12-9. 
Mitchison, D., \& Mond, J. (2015). Epidemiology of eating disorders, eating disordered behaviour, and body image disturbance in males: a narrative review. Journal of Eating Disorders, 3(1), 20. doi:10.1186/s40337-015-0058-y

Núñez-Navarro, A., Agüera, Z., Krug, I., Jiménez-Murcia, S., Sánchez, I., Araguz, N., ... Fernández-Aranda, F. (2012). Do men with eating disorders differ from women in clinics, psychopathology and personality? European Eating Disorder Review, 20(1), 2331. doi:10.1002/erv.1146

Sauchelli, S., Jiménez-Murcia, S., Sánchez, I., Riesco, N., Custal, N., Fernández-García, J. C., ... Fernández-Aranda, F. (2016). Orexin and sleep quality in anorexia nervosa: Clinical relevance and influence on treatment outcome. Psychoneuroendocrinology, 65, 102-108. doi:10.1016/j.psyneuen.2015.12.014

Shingleton, R., Thompson-brenner, H., Thompson, D. R., Pratt, E. M., \& Franko, D. L. (2015). Gender differences in clinical trials of binge eating disorder: An analysis of aggregated data. J Consult Clin Psychol, 83(2), 382-386. doi:10.1037/a0038849

Shu, C. Y., Limburg, K., Harris, C., Mccormack, J., Hoiles, K. J., Hamilton, M. J., \& Watson, H. J. (2015). Clinical presentation of eating disorders in young males at a tertiary setting. Journal of Eating Disorders, 3:39. doi:10.1186/s40337-015-0075-х

Steward, T., Mestre-Bach, G., Agüera, Z., Granero, R., Martín-Romera, V., Sánchez, I., ... Fernández-Aranda, F. (2016). Enduring Changes in Decision Making in Patients with Full Remission from Anorexia Nervosa. European Eating Disorders Review, 24(6), 
523-527. doi:10.1002/erv.2472

Støving, R. K., Andries, A., Brixen, K., Bilenberg, N., \& Hørder, K. (2011). Gender differences in outcome of eating disorders: A retrospective cohort study. Psychiatry Research, 186, 362-366. doi:10.1016/j.psychres.2010.08.005

Strober, M., Freeman, R., Lampert, C., Diamond, J., Teplinsky, C., \& DeAntonio, M. (2006). Are there gender differences in core symptoms, temperament, and short-term prospective outcome in anorexia nervosa? The International Journal of Eating Disorders, 39(7), 570-5. doi:10.1002/eat.20293

Strother, E., Lemberg, R., Stanford, S. C., \& Turberville, D. (2012). Eating disorders in men: Underdiagnosed, undertreated, and misunderstood. Eating Disorders, 20(5), 346-355. doi:10.1080/10640266.2012.715512

Thapliyal, P., \& Hay, P. J. (2014). Treatment experiences of males with an eating disorder: A systematic review of qualitative studies. Translational Developmental Psychiatry, 1, 19. doi:10.3402/tdp.v2.25552

Ulfvebrand, S., Birgegård, A., Norring, C., Högdahl, L., \& von Hausswolff-Juhlin, Y. (2015). Psychiatric comorbidity in women and men with eating disorders results from a large clinical database. Psychiatry Research, 230(2), 294-299. doi:10.1016/j.psychres.2015.09.008

Weltzin, T. E., Bean, P., Klosterman, E., Lee, H.-J., \& Welk-Richards, R. (2015). Sex differences in the effects of residential treatment on the quality of life of eating disorder 
patients. Eating and Weight Disorders, 20(3), 301-310. doi:10.1007/s40519-014-0162-z

Weltzin, T. E., Cornella-Carlson, T., Fitzpatrick, M. E., Kennington, B., Bean, P., \& Jefferies, C. (2012). Treatment issues and outcomes for males with eating disorders. Eating Disorders, 20(5), 444-59. doi:10.1080/10640266.2012.715527

Weltzin, T. E., Weisensel, N., Franczyk, D., Burnett, K., Klitz, C., \& Bean, P. (2005). Eating disorders in men: update. The Journal of Men's Health \& Gender, 2(2), 186-193. doi:10.1016/j.jmhg.2005.04.008

Woodside, D. B., Bulik, C. M., Thornton, L., Klump, K. L., Tozzi, F., Fichter, M. M., ... Kaye, W. H. (2004). Personality in men with eating disorders. Journal of Psychosomatic Research, 57(3), 273-278. 
Table 1. Sample description at baseline

\begin{tabular}{|c|c|c|c|c|c|c|}
\hline & Wome & $n=131$ & Men & $\eta=131$ & & \\
\hline Categorical variables & $\mathrm{n}$ & percent. & $\mathrm{n}$ & percent. & $\chi^{2}$ & $p$ \\
\hline Civil status & 102 & 77.9 & 98 & $74.8 \%$ & 1.41 & .493 \\
\hline Married - partner & 25 & $19.1 \%$ & 25 & $19.1 \%$ & & \\
\hline Divorced - separated & 4 & $3.1 \%$ & 8 & $6.1 \%$ & & \\
\hline Education level & 46 & $35.1 \%$ & 58 & $44.3 \%$ & 2.626 & .269 \\
\hline econdary & 64 & $48.9 \%$ & 52 & $39.7 \%$ & & \\
\hline$=$ University & 21 & $16.0 \%$ & 21 & $16.0 \%$ & & \\
\hline Employment status & 45 & $34.4 \%$ & 37 & $28.2 \%$ & 1.14 & .286 \\
\hline nployed & 86 & $65.6 \%$ & 94 & $71.8 \%$ & & \\
\hline Quantitative varia & Mean & SD & Mean & SD & $\mathrm{t}$ & $p$ \\
\hline Age (years-old) & 27.21 & 9.11 & 26.72 & 9.18 & 0.43 & .670 \\
\hline Age of onset (years-old) & 20.25 & 7.60 & 20.64 & 8.98 & 0.35 & .725 \\
\hline Disorder duration (years) & 6.73 & 6.38 & 6.27 & 6.04 & 0.43 & .669 \\
\hline Body mass index (kg/m2) & 22.07 & 6.88 & 23.49 & 7.99 & 1.50 & .135 \\
\hline Frequency vomits (per week) & 4.17 & 7.22 & 4.28 & 8.43 & 0.12 & .908 \\
\hline Frequency binges (per week) & 3.38 & 5.58 & 3.07 & 5.99 & 0.43 & .669 \\
\hline Frequency laxatives (per week) & 2.18 & 6.35 & 0.61 & 2.44 & 2.55 & .011 \\
\hline EDI-2: total score & 97.55 & 33.30 & 74.83 & 39.99 & 4.77 & $<.001$ \\
\hline
\end{tabular}

Note. SD: standard deviation. Bold: significant comparison (.05). 
Table 2. Comparison of treatment outcome between men and women with eating disorders

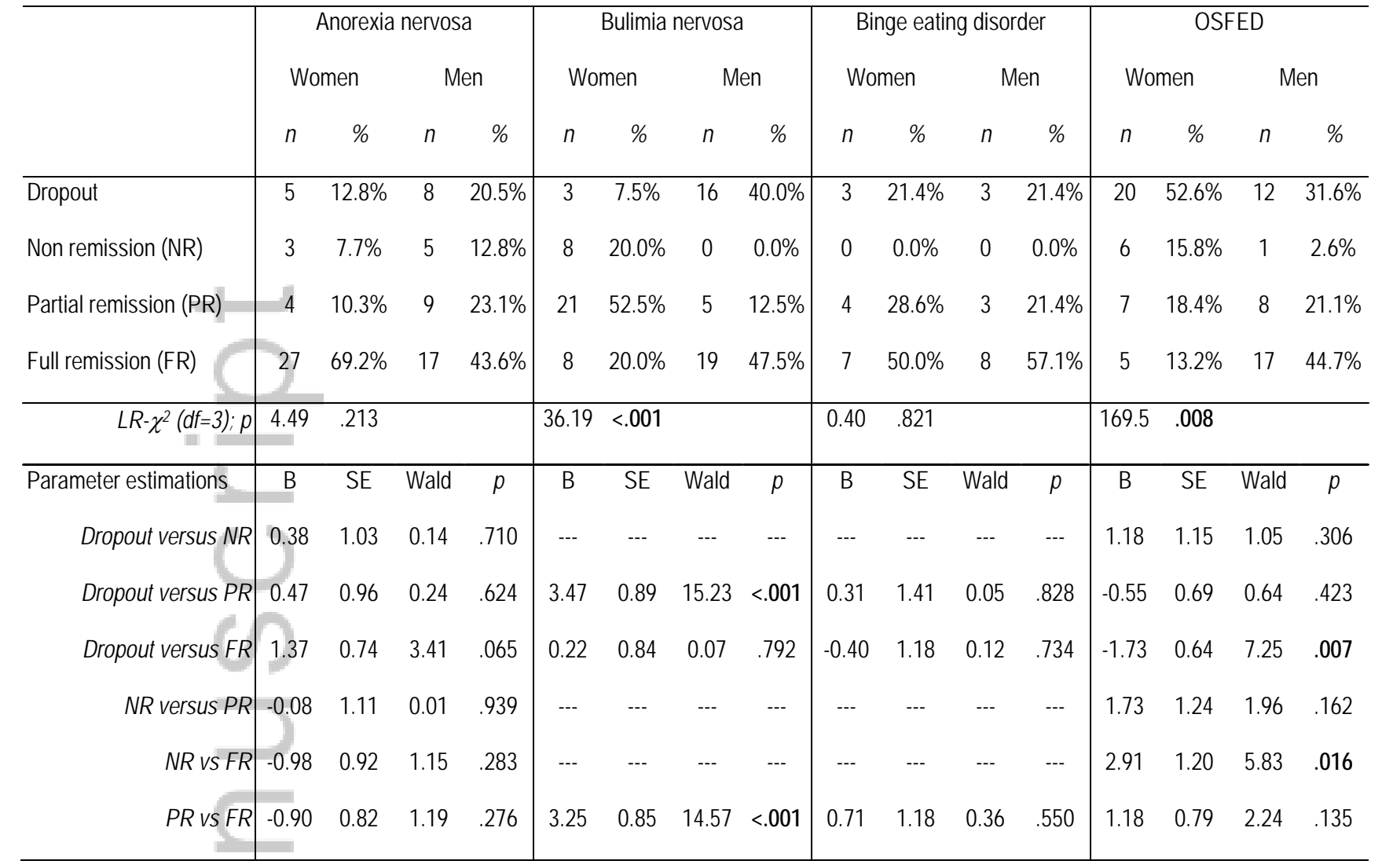

Note. Results obtained in multinomial regression adjusted for ED severity (EDI-2 total score). OSFED: other specified feeding or eating disorder. $\operatorname{LR}\left(\chi^{2}\right)$ : likelihood ratio chi-square test; $d f$ : degrees of freedom. Bold: significant comparison (.05). 
Table 3. Predictive models of therapy outcomes: stepwise logistic regression

\begin{tabular}{|c|c|c|c|c|c|c|c|c|c|c|c|c|c|}
\hline \multirow{3}{*}{ Dropout } & \multicolumn{6}{|c|}{ Women } & & \multicolumn{6}{|c|}{ Men } \\
\hline & \multirow[t]{2}{*}{ B } & \multirow[t]{2}{*}{ SE } & \multirow[t]{2}{*}{$p$} & \multirow[t]{2}{*}{ OR } & \multicolumn{2}{|c|}{$95 \% \mathrm{Cl}-\mathrm{OR}$} & & \multirow[t]{2}{*}{ B } & \multirow[t]{2}{*}{ SE } & \multirow[t]{2}{*}{$\mathrm{p}$} & \multirow[t]{2}{*}{ OR } & \multicolumn{2}{|c|}{$95 \% \mathrm{Cl}-\mathrm{OR}$} \\
\hline & & & & & & & Dropout & & & & & & \\
\hline Constant & -3.483 & 1.948 & .074 & 0.03 & & & Constant & 1.034 & 2.199 & .638 & 2.81 & & \\
\hline Covariate: ED diagnosis & & & .001 & & & & Covariate: ED diagnosis & & & .298 & & & \\
\hline AN vs OSFED & .965 & 0.621 & .002 & 0.14 & 0.04 & 0.47 & AN vs OSFED & -0.655 & 0.712 & .358 & 0.52 & 0.13 & 2.10 \\
\hline BN vs OSFED & 2.786 & 0.845 & .001 & 0.06 & 0.01 & 0.32 & $B N$ vs OSFED & 0.701 & 0.584 & .230 & 2.02 & 0.64 & 6.33 \\
\hline BED vs OSFED & -2.090 & 1.166 & .073 & 0.12 & 0.01 & 1.22 & BED vS OSFED & -0.015 & 0.914 & .987 & 0.99 & 0.16 & 5.91 \\
\hline Novelty seeking & 0.036 & 0.018 & .040 & 1.04 & 1.00 & 1.07 & Age (years-old) & -0.070 & 0.035 & .043 & 0.93 & 0.87 & 1.00 \\
\hline \multirow[t]{3}{*}{ Fitting: $H-L ; N R^{2} ; A \cup C$} & .948 & .344 & .819 & & & & Novelty seeking & 0.042 & 0.016 & .008 & 1.04 & 1.01 & 1.08 \\
\hline & & & & & & & Reward dependence & -0.045 & 0.017 & .007 & 0.96 & 0.92 & 0.99 \\
\hline & & & & & & & Fitting: H-L; NR2; AUC & .735 & .253 & .751 & & & \\
\hline Full remission & & & & & & & Full remission & & & & & & \\
\hline Constant & 1.768 & 2.056 & .390 & 5.86 & & & Constant & 0.304 & 1.564 & .846 & 1.36 & & \\
\hline Covariate: ED diac & & & .001 & & & & Covariate: ED diagnosis & & & .393 & & & \\
\hline AN vs OSFED & 3.142 & 0.732 & .001 & 23.15 & 5.51 & 97.24 & AN vs OSFED & 0.056 & 0.514 & .914 & 1.06 & 0.39 & 2.90 \\
\hline BN vs OSFED & 50 & 0.839 & .676 & 1.42 & 0.27 & 7.35 & $B N$ vs OSFED & 0.464 & 0.498 & .351 & 1.59 & 0.60 & 4.22 \\
\hline BED vs OSFED & 2.890 & 0.993 & .004 & 17.99 & 2.57 & 12.60 & BED vs OSFED & 1.120 & 0.698 & .108 & 3.06 & 0.78 & 12.03 \\
\hline Novelty seeking & -0.034 & 0.018 & .047 & 0.97 & 0.93 & 0.99 & Novelty seeking & -0.029 & 0.013 & .022 & 0.97 & 0.95 & 1.00 \\
\hline \multirow{2}{*}{\multicolumn{2}{|c|}{ Fitting: H-L; NR2; AUC .507}} & .333 & .822 & & & & Persistence & 0.021 & 0.010 & .030 & 1.02 & 1.00 & 1.04 \\
\hline & & & & & & & Fitting: H-L; NR2; $A \cup C$ & .098 & .119 & .716 & & & \\
\hline
\end{tabular}

Note. ED: eating disorder. AN: anorexia nervosa. BN: bulimia nervosa. OSFED: other specified feeding or eating disorder.

$\mathrm{H}-\mathrm{L}$ : Hosmer-Lemeshow test. N-R ${ }^{2}$ : Nagelkerke's pseudo- $\mathrm{R}^{2}$ coefficient. AUC: area under the ROC curve. 
Figure 1. Cumulate survival functions of the rate of dropout.

Note. Comparison by sex obtained in Cox-regression; adjusted for EDI-2 total score.

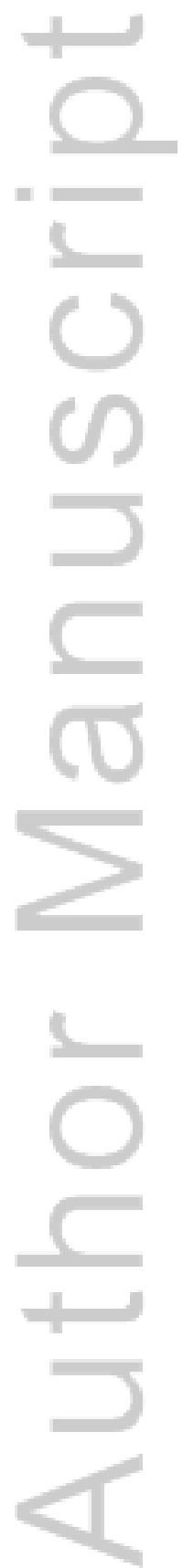

This article is protected by copyright. All rights reserved. 


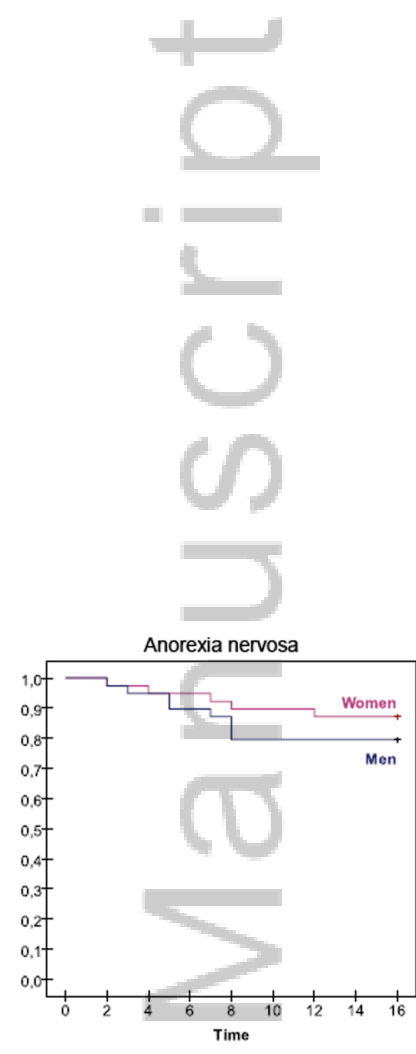
Wald $-x^{2}=2.26, d f=1, p=.132$ (2.
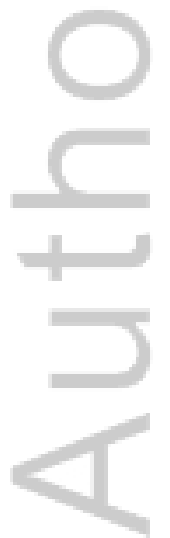

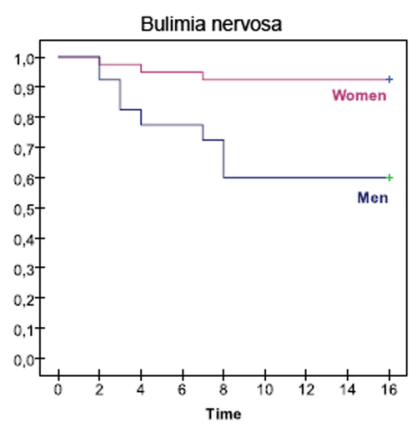

Wald $-\chi^{2}=7.57, d f=1, p=.006$

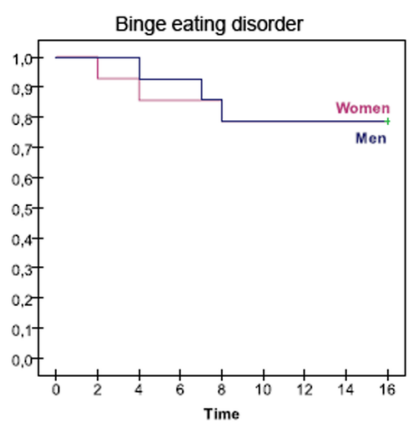

Wald $-\chi^{2}=0.47, d f=1, p=.829$

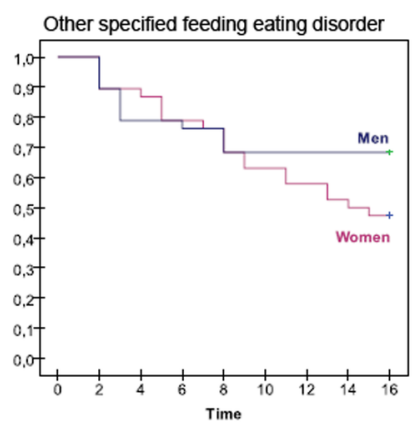

Wald $-\chi^{2}=2.02, d f=1, p=.155$

ERV_2519_F1.TIF

This article is protected by copyright. All rights reserved. 


\section{University Library}

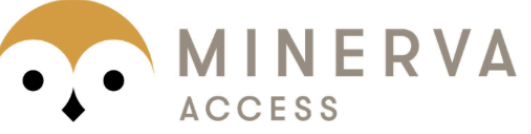

A gateway to Melbourne's research publications

Minerva Access is the Institutional Repository of The University of Melbourne

\section{Author/s:}

Aguera, Z;Sanchez, I;Granero, R;Riesco, N;Steward, T;Martin-Romera, V;Jimenez-Murcia, S;Romero, X;Caroleo, M;Segura-Garcia, C;Manuel Menchon, J;Fernandez-Aranda, F

Title:

Short-Term Treatment Outcomes and Dropout Risk in Men and Women with Eating Disorders

Date:

2017-07-01

\section{Citation:}

Aguera, Z., Sanchez, I., Granero, R., Riesco, N., Steward, T., Martin-Romera, V., JimenezMurcia, S., Romero, X., Caroleo, M., Segura-Garcia, C., Manuel Menchon, J. \& FernandezAranda, F. (2017). Short-Term Treatment Outcomes and Dropout Risk in Men and Women with Eating Disorders. EUROPEAN EATING DISORDERS REVIEW, 25 (4), pp.293-301. https://doi.org/10.1002/erv.2519.

Persistent Link:

http://hdl.handle.net/11343/292869 